\title{
Endoscopic ultrasound-guided drainage of a right liver abscess with a self-expandable metallic stent
}

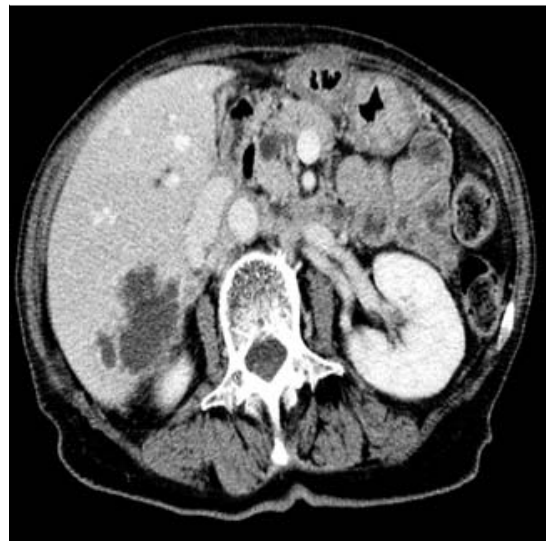

Fig. 1 Axial computed tomographic scan showing a liver abscess in the right hepatic lobe of an 81-year-old woman presenting with fever and abdominal pain.

Percutaneous drainage is one of the first options for the treatment of liver abscesses [1]. However, this method has several limitations, such as the requirement for external drainage and the risk for self-removal of the tube. On the other hand, endoscopic ultrasound (EUS)-guided drainage of liver abscesses overcomes both of these limitations. In addition, EUS-guided liver abscess drainage with a self-expandable metallic stent (SEMS) has a greater effect than percutaneous drainage, and leakage of the infected fluid is not likely to occur.

To date, only a few cases of EUS-guided liver abscess drainage with a SEMS have been reported [2]. Furthermore, EUSguided drainage of an abscess in the right hepatic lobe has not previously been reported. Herein, we describe our technique for performing EUS-guided drainage of an abscess of the right hepatic lobe with a SEMS.

An 81-year old woman was admitted to our hospital with fever and abdominal pain. Computed tomography revealed a liver abscess with a maximum diameter of $64 \mathrm{~mm}$ in the right hepatic lobe parenchyma ( $\bullet$ Fig. 1, Fig. 2). The patient had previously undergone metallic stent placement in the right hepatic bile duct because of unresectable cholangiocarcinoma. In addition, she had dementia; therefore, to avoid the risk for self-removal of the tube, we selected a transluminal approach with EUS.

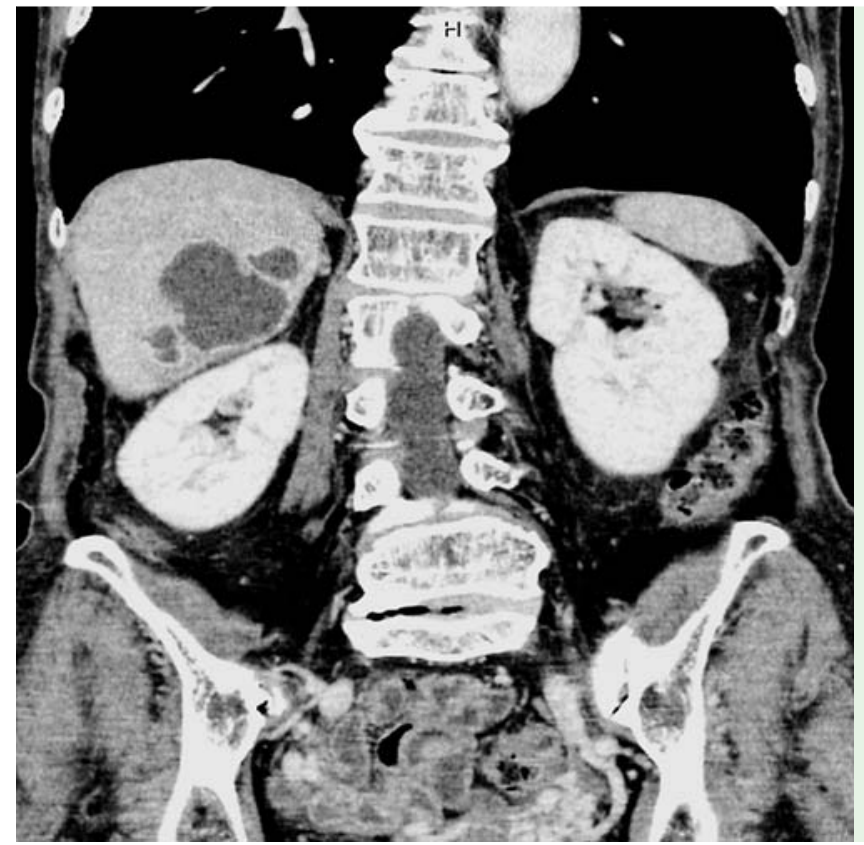

Fig. 2 Coronal computed tomographic scan of the liver abscess.

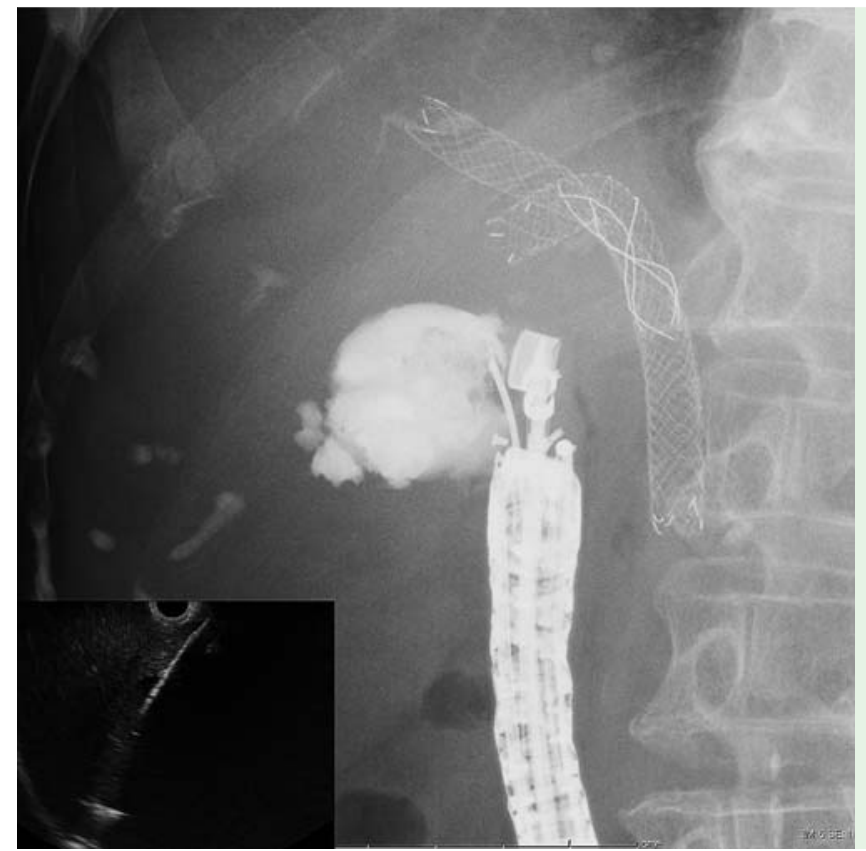

Fig. 3 The liver abscess was punctured with a 19-gauge fineneedle aspiration needle, and contrast medium was injected. Inset Endoscopic ultrasound-guided image showing a huge liver abscess.

First, the echoendoscope was advanced into the duodenum, and with counterclockwise rotation the right hepatic lobe was visualized. We punctured the liver abscess with a 19-gauge fine-needle aspiration needle (Medi-Globe $\mathrm{GmbH}$, Rosenheim, Germany), and the infected fluid was aspirated. Next, contrast medium was injected ( $\bullet$ Fig. 3), and a 0.025-inch guide- wire (VisiGlide; Olympus Medical Systems, Tokyo, Japan) was inserted. After the fistula had been dilated with a 4-mm Hurricane Balloon Dilatation Catheter (Boston Scientific, Tokyo, Japan), the stent delivery system was inserted. Finally, we successfully placed a fully covered SEMS (Bonastent, $10 \mathrm{~mm} \times 10 \mathrm{~cm}$; Standard SciTech, Seoul, Korea) from the liver abscess 


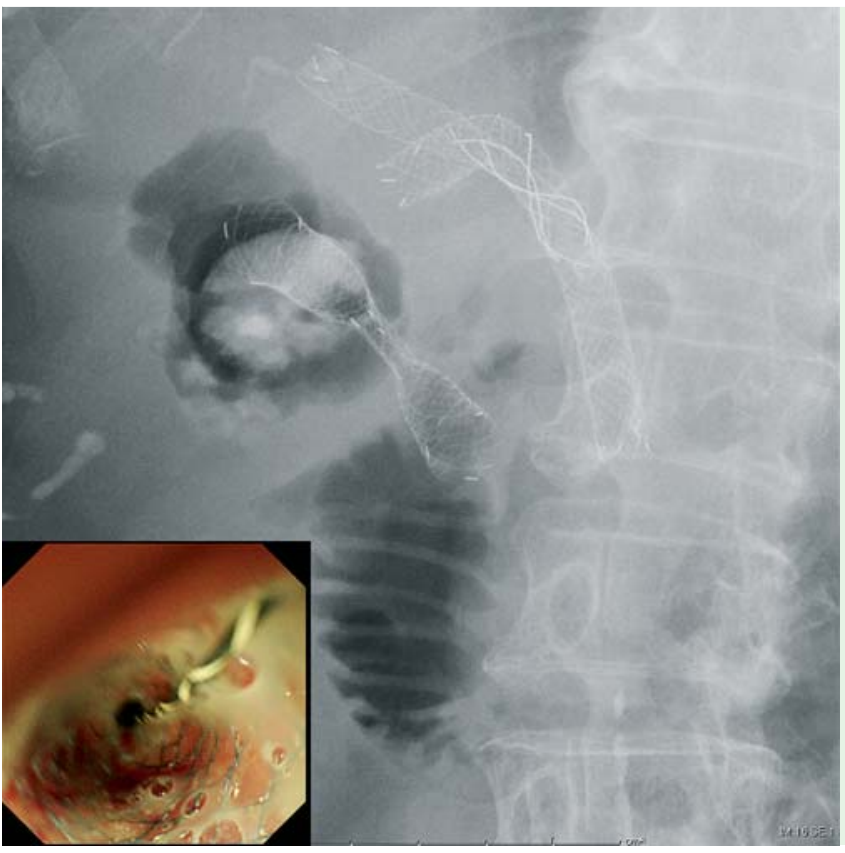

Fig.4 A fully covered self-expandable metallic stent was successfully placed from the liver abscess to the duodenum.

Inset $\mathrm{A}$ massive amount of infected material seen on the endoscopic image.

to the duodenum ( $\bullet$ Fig.4, $\bullet$ Video 1 ). The treatment resulted in a decrease in the size of the liver abscess, and the patient was discharged without any adverse events. EUS-guided liver abscess drainage has the potential to become the first-line method for draining liver abscesses because it can be used even for abscesses of the right hepatic lobe, as in the present case.

\section{Endoscopy_UCTN_Code_TTT_1AS_2AG}

\section{Competing interests: None}

Takeshi Ogura', Wataru Takagi', Saori Onda ${ }^{1}$, Daisuke Masuda ${ }^{1}$, Masayuki Kitano², Akira Imoto', Kazuhide Higuchi ${ }^{1}$

1 2nd Department of Internal Medicine, Osaka Medical College, Osaka, Japan

${ }^{2}$ Department of Gastroenterology and Hepatology, Kinki University Faculty of Medicine, Osaka-Sayama, Japan

\section{References}

1 Bertel CK, van Heerden JA, Sheedy PF 2nd. Treatment of pyogenic hepatic abscess. Surgical vs percutaneous drainage. Arch Surg 1986; 121: 554-558

2 Alcaide N, Vargass-Garcia AL, de la SermaHiguera $C$ et al. EUS-guided drainage of liver abscess by using a lumen-apposing metal stent (with video). Gastrointest Endosc 2013; 78: 941 -942; discussion 942

\section{Bibliography}

Dol http://dx.doi.org/

10.1055/s-0034-1392632

Endoscopy 2015; 47: E397-E398

(c) Georg Thieme Verlag KG

Stuttgart · New York

ISSN 0013-726X

\section{Corresponding author}

\section{Takeshi Ogura, MD}

2nd Department of Internal Medicine

Osaka Medical College

2-7 Daigakuchou

Takatsukishi

Osaka 569-8686

Japan

Fax: +81-72-684-6532

The liver abscess was punctured with a 19-gauge fine-needle aspiration needle, and contrast medium was injected. Then, a guidewire was inserted into the liver abscess. First, an endoscopic retrograde cholangiopancreatography (ERCP) catheter was inserted to dilate the fistula. Next, balloon dilation was performed. Finally, a stent was successfully placed from the liver abscess to the duodenum. 